OUTP-9403; FTUAM94/7

\title{
Fermion masses and mixing angles from gauge symmetries.
}

\author{
Luis Ibáñez, \\ Departamento de Fisica Teórica \\ Universidad Autónoma de Madrid \\ Cantoblanco, 28034 Madrid \\ and \\ Graham G. Ross*, \\ Department of Physics, \\ Theoretical Physics, \\ University of Oxford, \\ 1 Keble Road, \\ Oxford OX1 3NP
}

\begin{abstract}
The structure of the quark and lepton masses and mixing angles provides one of the few windows we have on the underlying physics generating the Standard Model. In an attempt to identify the underlying symmetry group we look for the simplest gauge extension of the SUSY standard model capable of generating the observed structure. We show that the texture structure and hierarchical form found in the (symmetric) quark and lepton mass matrices follows if one extends the gauge group of the standard model to include an horizontal $U(1)$ gauge factor, constrained by the need for anomaly cancellation. This $U(1)$ symmetry is spontaneously broken slightly below the unification/string scale leaving as its only remnant the observed structure of masses and mixings. Anomaly cancellation is possible only in the context of superstring theories via the Green Schwarz mechanism with $\sin ^{2}\left(\theta_{W}\right)=3 / 8$.
\end{abstract}

${ }^{*}$ SERC Senior Fellow 


\section{Introduction}

There has been considerable success explaining the parameters of the Standard Model in the framework of a supersymmetric extension of the model with a stage of unification. The measured values of the gauge couplings are consistent with their normal unification values with a unification scale of $\mathrm{O}\left(10^{16} \mathrm{GeV}\right)$ provided, at low energies $\leq O\left(10^{3} \mathrm{GeV}\right)$, the Standard Model spectrum is extended to that of the minimal supersymmetric model (the MSSM) [1]. In addition the pattern (and magnitude) of spontaneous breakdown of the Standard Model follows naturally from the structure of radiative corrections in the MSSM provided there is some unification of the supersymmetry breaking masses at the unification scale 11, 2]. This simplicity in the parameters of the (supersymmetric) Standard Model a high scales appears to extend to some of the couplings involved in determining the fermion masses. The measured values of the bottom quark and the $\tau$ lepton are consistent with their equality at the unification scale 3, 4]. Further the mixing angles and masses have values consistent with the appearance of "texture" zeros in the mass matrix [5, 6, 7, 8, such texture zeros indicating the appearance of additional symmetries beyond the Standard Model.

In this paper we will explore the possibility that at least some of the symmetries giving rise to this texture structure are new gauge symmetries. Given the success of the MSSM we will look for the minimal extension of the MSSM able to generate a Yukawa structure which is phenomenologically viable. We consider only the case of symmetric mass matrices' for this allows us to determine the structure of the mass matrices with texture zeros and hence provides us with a definite starting point for the search for new symmetries. Surprisingly we find that the simplest possibility in which one extends the Standard Model to include an extra $U(1)$ symmetry is already sufficient to generate an interesting texture structure. However, this extra $U(1)$ has a stringy nature: by itself it is anomalous and its anomaly is cancelled by a Green- Schwarz[9] mechanism coming from an underlying string theory. Remarkably this cancellation only works if the Kac Moody level structure associated with the Standard Model gauge groups is such that the gauge couplings have their normal unification values leading to the successful unification predictions noted above.

\section{Quark mass matrices.}

The structure of the quark mass matrices is not directly measured because the charged weak current only tells us about the mixing in the left-handed sector. If, however, one adds the requirement of symmetry relating the left to the right sector, the extraction of the mass matrices from data becomes feasible 11. In

\footnotetext{
${ }^{1}$ This restriction is also motivated by our desire to identify the maximally symmetric possibility consistent with observation, and suggests there is a further left-right symmetry at high scales.
} 


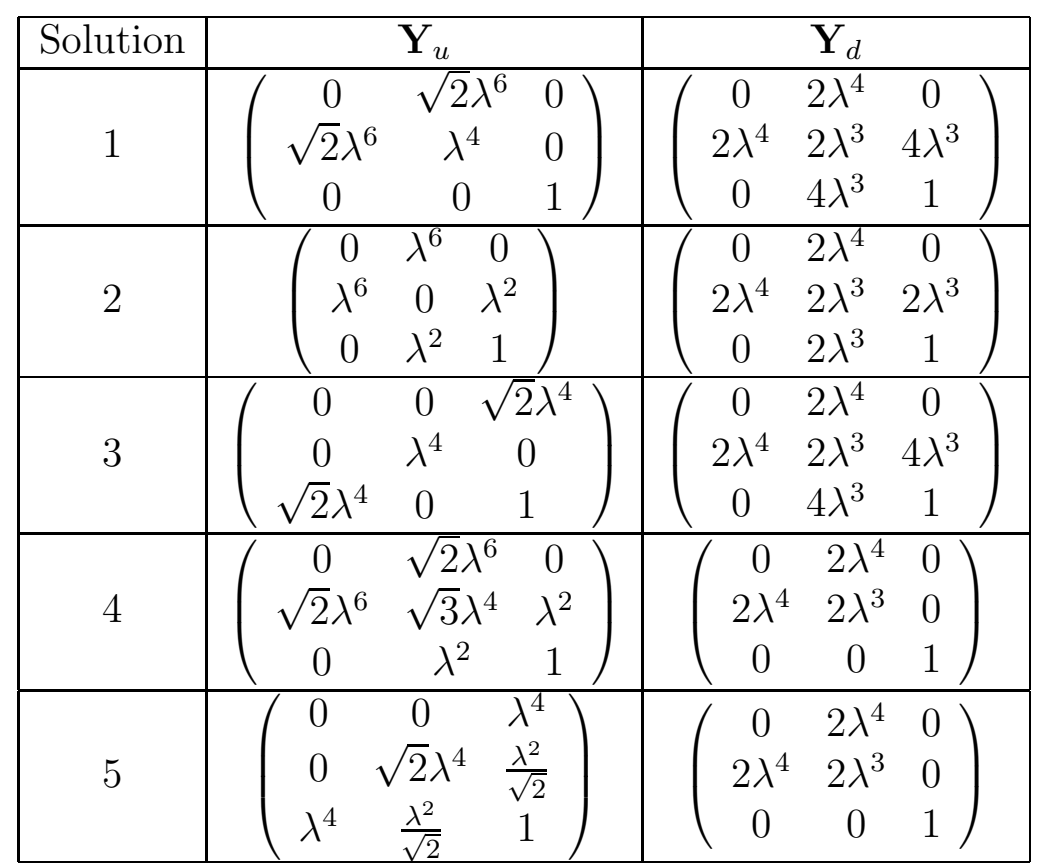

Table 1: Approximate forms for the symmetric textures of quark masses.

\begin{tabular}{|c|c|c|c|c|}
\hline Solution & $\frac{m_{c}}{m_{t}}$ & $\frac{m_{u}}{m_{c}}$ & $V_{c b}$ & $\frac{V_{u b}}{V_{c b}}$ \\
\hline "Experiment" & $0.006-0.01$ & $0.003-0.005$ & $0.02-0.05$ & $0.05-0.13$ \\
\hline 1 & $6.710^{-3}$ & 0.0046 & $610^{-2}$ & 0.068 \\
\hline 2 & $6.710^{-3}$ & 0.0023 & $3.810^{-2}$ & 0.0484 \\
\hline 3 & $6.710^{-3}$ & 0.0046 & $610^{-2}$ & 0.078 \\
\hline 4 & $4.910^{-3}$ & 0.0087 & $6.810^{-2}$ & 0.040 \\
\hline 5 & $6.110^{-3}$ & 0.003 & $4.810^{-2}$ & 0.068 \\
\hline
\end{tabular}

Table 2: Predictions following from the five symmetric texture solutions using $\lambda=0.22$. All solutions give $V_{u s}=0.22$ and $\frac{m_{d}}{m_{s}}=0.05$ and $\frac{m_{s}}{m_{b}}=0.03$, in agreement with the experimental results $\frac{m_{d}}{m_{s}}=0.04-0.067$ and $\frac{m_{s}}{m_{b}}=0.03-0.07[10]$.

particular we wish to consider the possibility that the mass matrices are leftright symmetric i.e., invariant under the $Z_{2}$ exchange $q_{R} \leftrightarrow q_{L}$. In this case the experimental measurements of quark masses and mixing angles may be used to determine the mass matrices with the maximum number of texture zeros. As we shall discuss, given the form of these matrices allows us to determine whether additional symmetries are capable of generating their structure and hence of giving the observed quark masses and mixing angles.

The symmetric mass matrices with five texture zeros are given in Table 11]1]. In Table $11 \lambda$ is an expansion parameter, essentially the $(1,2)$ matrix element of the CKM matrix. We should stress that these are approximate forms in the sense

\footnotetext{
${ }^{2}$ Although six texture zeros is the maximum possible, no examples were found consistent with the masses and mixing angles.
} 
that the texture zeros should be interpreted as being zero only up to the order that does not significantly change the masses and mixing angles. For example Solutions 1, 2 and 4 all have texture zeros in the $(1,1)$ and $(1,3)$ positions of both $Y_{u}$ and $Y_{d}$. This leads to the predictions 11

$$
\begin{aligned}
\left|\frac{V_{u b}}{V_{c b}}\right| & =\sqrt{\frac{m_{u}}{m_{c}}} \\
\left|V_{u s}\right| & =\left(\frac{m_{d}}{m_{s}}+\frac{m_{u}}{m_{c}}+2 \sqrt{\frac{m_{d} m_{u}}{m_{s} m_{c}}} \cos \phi^{\prime}\right)^{\frac{1}{2}}
\end{aligned}
$$

where $\phi^{\prime}$ is a phase related to the phases (not displayed) of the non-zero matrix elements of Table1. To preserve these phenomenologically successful relations requires $Y_{u}(1,1)<O\left(\lambda^{8}\right), Y_{u}(1,3)<O\left(\lambda^{4}\right), Y_{d}(1,1)<O\left(\lambda^{4}\right), Y_{d}(1,3)<O\left(\lambda^{2}\right)$. On the other hand the $(2,2)$ zero in $Y_{u}$ in Solution 2 is not so critical and an entry of $O\left(\lambda^{4}\right)$ is acceptable. We shall see that this is important in the models presented below which generate approximate texture zeros.

The structure of Table 1 strongly suggests an underlying chiral symmetry broken by terms of $O(\lambda)$. In the limit the symmetry is exact only the third generation is massive and all mixing angles are zero. Symmetry breaking terms gradually fill in the mass matrices generating an hierarchy of mass scales and mixing angles $17,12,13,14,15$. In the next section we address the question

whether a spontaneously broken gauge symmetry is capable of generating this structure.

\section{Gauging a family $U(1)$ symmetry}

We wish to discuss the possibility that this structure results from the simplest possible gauge extension of the Standard Model namely an abelian horizontal gauge factor acting on the family or generation indices. Without loss of generality we write the $\mathrm{U}(1)$ in the form

$$
U(1)=U(1)_{F D}+U(1)_{F I}
$$

where $U(1)_{F D}$ is a family dependent symmetry, by definition acting only on the quarks and leptons and $U(1)_{F I}$ is a family independent symmetry. If the fermion mass matrix is to be symmetric $U(1)_{F D}$ must act the same way on left- and right handed components while $U(1)_{F I}$ is not constrained. It proves convenient to consider first the structure of $U(1)_{F D}$ as it determines the relative magnitudes of the matrix elements within a single (up, down or lepton) mass matrix.

The $U(1)_{F D}$ charges of the MSSM states are given in Table 3 . The condition of symmetric matrices requires that all quarks(leptons) of the same i-th generation transform with the same charge $\alpha_{i}\left(a_{i}\right)$. Through a choice of $U(1)_{F I}$ we may 


\begin{tabular}{|c|ccccccc|}
\hline & $\mathrm{Q}$ & $\mathrm{u}$ & $\mathrm{d}$ & $\mathrm{L}$ & $\mathrm{e}$ & $H_{2}$ & $H_{1}$ \\
\hline$U(1)_{F D}$ & $\alpha_{i}$ & $\alpha_{i}$ & $\alpha_{i}$ & $a_{i}$ & $a_{i}$ & $-2 \alpha_{1}$ & $w \alpha_{1}$ \\
\hline
\end{tabular}

Table 3: $U(1)_{F D}$ symmetries. $w=-2$ corresponds to up-down symmetric matrices

make $U(1)_{F D}$ traceless without any loss of generality. Thus $\alpha_{3}=-\left(\alpha_{1}+\alpha_{2}\right)$ and $a_{3}=-\left(a_{1}+a_{2}\right)$.

With this we may now consider the constraints on the Yukawa couplings generating the mass matrices. The $U(1)_{F D}$ charge of the quark-antiquark pair has the form

$$
\left(\begin{array}{ccc}
-2\left(\alpha_{1}+\alpha_{2}\right) & -\alpha_{1} & -\alpha_{2} \\
-\alpha_{1} & 2 \alpha_{2} & \alpha_{1}+\alpha_{2} \\
-\alpha_{2} & \alpha_{1}+\alpha_{2} & 2 \alpha_{1}
\end{array}\right)
$$

This matrix neatly summarises the allowed Yukawa couplings for a Higgs boson coupling in a definite position should have charge minus that shown for the relevant position.

For the leptons we have a similar structure of lepton-antilepton charges

$$
\left(\begin{array}{ccc}
-2\left(a_{1}+a_{2}\right) & -a_{1} & -a_{2} \\
-a_{1} & 2 a_{2} & a_{1}+a_{2} \\
-a_{2} & a_{1}+a_{2} & 2 a_{1}
\end{array}\right)
$$

We will now consider in turn the implications of this structure for the up and down quark and lepton mass matrices respectively.

\section{The up quark mass matrix.}

We first consider the up quark mass matrix. We assume that the light Higgs, $\mathrm{H}_{2}$, has $U(1)$ charge so that only the $(3,3)$ renormalisable Yukawa coupling to $H_{2}$ is allowed. In this way only the $(3,3)$ element of the associated mass matrix will be non-zero as desired to reproduce the leading structure of Table 1. The remaining entries must be generated when the $U(1)$ symmetry is broken. Suppose singlet fields, $\theta, \bar{\theta}$, with $U(1)_{F D}$ charge $-1,+1$ respectively acquire equal vacuum expectation values (vevs) along a "D-flat' direction, spontaneously breaking this symmetry'3. After this breaking all entries in the mass matrix become non-zero. For example, the $(3,2)$ entry appears at $O\left(\epsilon^{\left|\alpha_{2}-\alpha_{1}\right|}\right)$ because $\mathrm{U}(1)$ charge conservation allows only a coupling $c^{c} t H_{2}\left(\theta / M_{2}\right)^{\alpha_{2}-\alpha_{1}}, \alpha_{2}>\alpha_{1}$ or $c^{c} t H_{2}(\bar{\theta} / M)^{\alpha_{1}-\alpha_{2}}, \alpha_{1}>\alpha_{2}$

\footnotetext{
${ }^{3}$ The spontaneous breaking of gauge symmetries at high scales in supersymmetric theories must proceed along such flat directions to avoid large vacuum energy contributions from Dterms.
} 
and we have defined $\epsilon=\left(\theta / M_{2}\right)$ where $M_{2}$ is the unification mass scale which governs higher dimension operators.

Further elements may be generated depending on the values of $\alpha_{1}$ and $\alpha_{2}$, giving the structure

$$
M_{u} \approx\left(\begin{array}{ccc}
\epsilon^{\left|-4 \alpha_{1}-2 \alpha_{2}\right|} & \epsilon^{\left|-3 \alpha_{1}\right|} & \epsilon^{\left|-\alpha_{2}-2 \alpha_{1}\right|} \\
\epsilon^{\left|-3 \alpha_{1}\right|} & \epsilon^{\left|2\left(\alpha_{2}-\alpha_{1}\right)\right|} & \epsilon^{\left|\alpha_{2}-\alpha_{1}\right|} \\
\epsilon^{\left|-\alpha_{2}-2 \alpha_{1}\right|} & \epsilon^{\left|\alpha_{2}-\alpha_{1}\right|} & 1
\end{array}\right)
$$

Note that an hierarchical structure immediately appears if $\epsilon$ is small. Further one already draw some general conclusions for we have

$$
M_{11}^{u} \simeq \frac{\left(M_{13}^{u}\right)^{2}}{M_{33}^{u}} ; \quad M_{22}^{u} \simeq \frac{\left(M_{23}^{u}\right)^{2}}{M_{33}^{u}}
$$

independently of what specific $U(1)_{F D}$ quantum numbers one is assuming. Thus different elements are related in a manner remarkably consistent with Solutions 1, 2 and 4 of Table 1; a texture zero in the $(1,3)$ position is correlated with a texture zero in the $(1,1)$ position.

The condition that such a zero occurs depends only on the ratio $\alpha_{2} / \alpha_{1}$. Remarkably, for the range $\alpha_{2} / \alpha_{1}>1$, there are texture zeros in the $(1,1)$ and $(1,3)$ positions just as required in Solutions 1, 2 or 4 of Table 11 1 ! As just noted the correlation between the $(1,3)$ and $(1,1)$ zeros is obvious from the first relation of eq(6).

It is very easy to find a simple choice of $\alpha_{2} / \alpha_{1}$ which even generates the correct order for the non-zero elements of one of the Solutions 1, 2 or 4 of Table 1. If one requires that the $(1,2)$ and $(2,3)$ matrix elements be in the ratio 3:1 (as needed for Solution 2 or 4 ) then $\alpha_{2}=2 \alpha_{1}$ and the up quark mass matrix has the form

$$
M_{u} \approx\left(\begin{array}{ccc}
\epsilon^{8} & \epsilon^{3} & \epsilon^{4} \\
\epsilon^{3} & \epsilon^{2} & \epsilon \\
\epsilon^{4} & \epsilon & 1
\end{array}\right)
$$

where we have used the freedom to set $\alpha_{1}=1$ through a redefinition of the parameter $\epsilon$ and $\alpha_{2}$ (i.e. $\left.\epsilon \rightarrow(\theta / M)^{\alpha_{1}}, \alpha_{2} \rightarrow \alpha_{2} / \alpha_{1}\right)$.

This is the structure of solutions 2 or 4 (and indeed 1) of Table 1 to the accuracy of the Table! The origin of this structure deserves some comment. The relative magnitudes of the $(1,2)$ and $(2,3)$ elements comes from our assumption about the relative magnitudes of $\alpha_{1}$ and $\alpha_{2}$. The assumption that these charges are quantised is quite reasonable if the $U(1)$ is embedded in some larger non-Abelian GUT or if it comes from a 4D superstring theory. As noted above the remaining structure, in particular the texture zeros in the $(1,1)$ and $(1,3)$ positions, are predicted by the anomaly cancellation condition which fixed the

\footnotetext{
${ }^{4}$ These are "zeros" in the sense discussed above that their values do not affect the masses or mixing angles in leading order.
} 
transformation properties of the first generation. Notice that, on the other hand, textures of type 3 or 5 would be difficult to obtain in the present approach. Thus the present simple approach gives us a hint on what type of generic textures to consider if we want them to be generated by a simple abelian family structure.

\subsection{The origin of the higher dimension couplings.}

It is appropriate to discuss, at this stage, how the non-renormalisable terms needed to fill in the mass matrix may arise. There are three possible sources. The first possibility is that a term such as $c^{c} t H_{2}(\theta / M)$ occurs at the string compactification level. In this case we expect the mass scale $M$ to be the string scale $M_{s}\left(\approx M_{\text {Planck }}\right)$. In addition there may be a systematic suppression of such terms for all higher dimension terms of this type are accompanied by a factor of the form $\exp (-a T)$ where a is a term-dependant constant and $\mathrm{T}$ is a moduli-field which sets the overall radius of compactification.

The second possible origin of the higher dimension terms is the mixing between light and heavy Higgs states. Consider a string compactification which in addition to $H_{1}$ and $H_{2}$, leaves additional Higgs multiplets $H_{1,2}^{a, b \ldots}, \bar{H}_{1,2}^{a, b \ldots}$ light. This is indeed what happens in many compactification schemes for, in addition to the three generations of quarks and leptons needed for a viable theory, there are additional vector-like pairs of quarks, leptons and Higgs fields. For example, in Calabi-Yau compactification, there are $\left(h_{2,1}-h_{1,1}\right)$ generations, where $h_{2,1}$ and $h_{1,1}$ are the Hodge numbers counting $(2,1)$ and $(1,1)$ forms and there are $h_{1,1}$ additional pairs of conjugate representations. Similarly there are usually additional light Higgs states in conjugate representations generated on compactification. Of course such additional states would be an embarrassment it they remained light at low scales but, being in conjugate representations, they may be expected to gain mass if the gauge symmetry is spontaneously broken after compactification through their coupling to the scalar field acquiring a vacuum expectation value. Thus, in any compactification scheme with a gauge group larger than that of the standard model, the states $H_{1,2}^{a, b}, \bar{H}_{1,2}^{a, b \ldots}$ may be expected to acquire masses, $M_{1,2}$ of the order of the breaking scale of the enlarged gauge group. However if there is more than one such breaking scale there may be more than one source of mass so that $M_{1}$ and $M_{2}$ may differ.

After the various stages of spontaneous breaking at the high scale, the Higgs state left light will be a combination of all the original Higgs fields carrying the same $S U(3) \otimes S U(2) \otimes U(1)$ gauge quantum numbers. In particular, due to the $<\theta>$ vev the light Higgs may carry a mixture of $\mathrm{U}(1)$ charges

$$
H_{1,2}^{\text {light }} \simeq H_{1,2}+\sum_{r=1}\left(H_{1,2}^{r} \frac{<\theta>^{r}}{M_{1,2}^{r}}+H_{1,2}^{-r} \frac{<\bar{\theta}>^{r}}{M_{1,2}^{r}}\right)
$$

where we have denoted by $H_{1,2}^{r}$ an Higgs field carrying $\mathrm{U}(1)$ charge r. Clearly $H_{2}^{\text {light }}$ will generate all elements of $M^{u}$; for example the $(3,2)$ matrix element will 
arise at $0\left(<\theta>/ M_{2}\right)$ where $M_{2}$ is the mass of the heavy $H_{2}^{1}$ field before the $<\theta>$ vev causes it to mix with $H^{\text {light }}$.

The third sources of the higher dimension terms in $m^{u}$ is through the mixing of quark $U(1)$ eigenstates in a manner analogous to that just discussed for the Higgs. In this case

$$
q^{l i g h t} \simeq q+\sum_{r=1} q_{r} \frac{<\theta>^{r}}{M_{q}^{r}}+q_{-r} \frac{<\bar{\theta}>^{r}}{M_{q}^{r}}
$$

where $M_{q}$ is the mass of the heavy quark state in the absence of the $\langle\theta\rangle$ vev. This will also generate non-zero entries for $m^{u}$; for example the $(3,2)$ matrix element will arise at $0\left(<\theta>/ M_{q}\right)$.

The question which contribution dominates is model dependent and depends on whether the appropriate $H_{1}^{r}$ or $q_{r}$ is left light after compactification, on the relative magnitudes of $M_{q}, M_{1}, M_{s}$ and on whether, as may happen, the $\theta$ couples only to the quarks or to the Higgs.

\subsection{The down quark mass matrix.}

We have seen that it is very easy to get an acceptable form for the up quark mass matrix using a very simple choice for $U(1)_{F D}$. We turn now to the question whether this is consistent with the structure required for the down quark mass matrix. Our requirement of a symmetric mass matrix together with $S U(2)_{L}$ immediately gives the $U(1)_{F D}$ charge structure of eq(3). The structure of the down quark mass matrix then depends only on the $U(1)$ charge of $H_{1}^{\text {light }}$. This will be constrained by further anomaly cancellation conditions, principally the $S U(2)^{2} U(1)$ condition, which we will discuss in Section 6. Here we first consider whether any $U(1)$ charge assignment for $H_{1}$ leads to an acceptable down quark mass matrix.

We focus on the interesting case $\alpha_{2}=2 \alpha_{1}$ which gave an acceptable up quark mass matrix. Assigning a $U(1)$ charge $w \alpha_{1}$ to $H_{1}$ f leads to the following form for the down quark mass matrix:

$$
M_{d}=\left(\begin{array}{ccc}
\bar{\epsilon}^{|-6+w|} & \bar{\epsilon}^{|-1+w|} & \bar{\epsilon}^{|-2+w|} \\
\bar{\epsilon}^{|-1+w|} & \bar{\epsilon}^{|4+w|} & \bar{\epsilon}^{|3+w|} \\
\bar{\epsilon}^{|-2+w|} & \bar{\epsilon}^{|3+w|} & \bar{\epsilon}^{|2+w|}
\end{array}\right)
$$

where $\bar{\epsilon}=\left(\frac{\langle\theta\rangle}{M_{1}}\right)$ and $M_{1}$ is the appropriate scale for these higher dimension terms (cf $M_{2}$ for in the case of $M_{u}$ ). Following the discussion of Section 4.1, if the dominant source of these terms is from string compactification then we expect $M_{1}=M_{2}=M_{s}$ and $\epsilon=\bar{\epsilon}$. The same is true if these terms arise due to quark mixing when $M_{1}=M_{2}=M_{q}$. However if they are due to Higgs mixing then strong violation may occur of the $S U(2)_{R}$ symmetry of the quark sector

\footnotetext{
${ }^{5}$ If $b^{c} b$ has a $U(1)_{F I}$ charge, $\delta$, we assume that $H_{1}$ has an additional charge - $\delta$.
} 
which was forced on us by our assumption of left- right- symmetry. The reason is because vectorlike pairs $H_{1,2}, \bar{H}_{1,2}$ left massless after compactification must acquire their mass via a stage of spontaneous breaking after compactification and this need not respect $S U(2)_{R}$. As a result $M_{1} \neq M_{2}$ and $\epsilon \neq \bar{\epsilon}$.

Again we see from eq(10) that an hierarchical structure for $m_{d}$ automatically results. As we will discuss this can be of an acceptable form. For example with $w=-2$ one gets a matrix with the same structure as eq(7)

$$
M_{d} \approx\left(\begin{array}{ccc}
\bar{\epsilon}^{8} & \bar{\epsilon}^{3} & \bar{\epsilon}^{4} \\
\bar{\epsilon}^{3} & \bar{\epsilon}^{2} & \bar{\epsilon} \\
\bar{\epsilon}^{4} & \bar{\epsilon} & 1
\end{array}\right)
$$

As we have discussed there is no reason why $\epsilon=\bar{\epsilon}$; if they are not there will be difference between the up and down mass matrices. For a suitable choice of $\bar{\epsilon}$ this can reproduce quite closely the second solution of Table 11. We will return to a detailed discussion of this possibility in the next Section.

Another interesting choice is $w=-3 / 2$ for which $m^{d}$ is given by

$$
M_{d} \approx \sqrt{\bar{\epsilon}}\left(\begin{array}{ccc}
\bar{\epsilon}^{7} & \bar{\epsilon}^{2} & \bar{\epsilon}^{3} \\
\bar{\epsilon}^{2} & \bar{\epsilon}^{2} & \bar{\epsilon} \\
\bar{\epsilon}^{3} & \bar{\epsilon} & 1
\end{array}\right)
$$

Although not of the form of any of the matrices in Table(1), this structure may be viable if the residual Yukawa couplings associated with each entry which are not displayed here and are implicitly assumed here to be of $O(1)$ are not all equal in magnitude. Note that eq(12) has the interesting implication that there is a suppression of $m_{b}$ relative to $m_{t}$ due to the overall factor of $\sqrt{\bar{\epsilon}}$. This factor, together with the difference in vevs of $H_{1,2}$ can describe why $m_{b}<<m_{t}$. In the case of $w=-2$ the difference between $m_{b}$ and $m_{t}$ must come entirely from the difference in $H_{1,2}$ vevs.

At this point an important remark is in order. If we try to do without $U(1)_{F I}$ in eq(2) anomaly cancellation requires the $U(1)_{F I}$ charge for the $H_{1}$ field to be $w=+2$ so that the $S U(2)^{2} U(1)_{F I}$ anomalies coming from the Higgs contribution cancel. In this case the form of the d-quark mass matrix is a disaster, giving

$$
M_{d}=\left(\begin{array}{ccc}
\bar{\epsilon}^{4} & \bar{\epsilon} & 1 \\
\bar{\epsilon} & \bar{\epsilon}^{6} & \bar{\epsilon}^{5} \\
1 & \bar{\epsilon}^{5} & \bar{\epsilon}^{4}
\end{array}\right)
$$

which is clearly not viable. Another obvious alternative to achieve cancellation of anomalies is to assume that the Higgs fields are neutral. But in this case one needs to set $\alpha_{1}=0$ and anomaly cancellation yields $\alpha_{2}=-\alpha_{3}$. These values in turn imply that the three generations would be degenerate in mass.

From this we conclude that the $U(1)_{F I}$ component is necessary. As we shall discuss in Section 6 it is then possible to find an anomaly free solution but only in

\footnotetext{
${ }^{6}$ This choice requires fractional charges $\pm 1 / 2$ for the $\theta, \bar{\theta}$ fields.
} 
a string theory in which the four-dimensional version of the Green-Schwarz (GS) mechanism cancels the anomalies.

\subsection{Phenomenology of the quark mass matrices.}

As we have seen the structures emerging from the requirement there be an additional abelian gauge symmetry can reproduce the hierarchical structure observed. The $U(1)$ symmetry by itself only determines the order of the matrix elements and if the constants of proportionality are chosen as in Solution 2 of Table 1 the masses and mixing angles are in agreement with experiment as may be seen in Table 4 (Note, as discussed in Section 2 that the $(2,2)$ entry of $Y_{u}$ in solution 2 is only zero to $O\left(\lambda^{2}\right)$, allowing this solution to be viable!). To go further it is necessary to determine the constants of proportionality in each matrix element. In this section we will explore whether any of the structures discussed above are consistent with a larger symmetry relating these constants.

As we discussed in Section 4.1, the higher dimension contributions may arise directly in the effective theory descending from the string, or indirectly, through mixing of the quarks and/or Higgs. Let us consider the case with $w=-2$. In this case the form of the up and down quark mass matrices is the same due to an effective $S U(2)_{L} \otimes S U(2)_{R}$ symmetry in their couplings forced on us by our assumption of left- right- symmetric mass matrices. In order to allow for a difference between up and down quark mass matrices we have argued that the mixing through the Higgs sector must dominate. In this case the expansion parameter for the up and down quark sector differs due to the different masses of the $H_{1}$ and $H_{2}$ fields triggered by the breaking of the $S U(2)_{R}$ symmetry. However we may still ask whether the underlying couplings, which determine the constants of proportionality, are related by the $S U(2)_{R}$ symmetry, revealing a more symmetric solution. Further relations between mass matrix elements require a model of the underlying physics generating the higher dimension operators and lie outside the scope of this paper.

Putting the constants of proportionality back in to the mass matrices and dropping small elements gives the form

$$
\begin{aligned}
M_{u} & \approx\left(\begin{array}{ccc}
0 & b \epsilon^{3} & 0 \\
b \epsilon^{3} & \epsilon^{2} & a \epsilon \\
0 & a \epsilon & 1
\end{array}\right) \\
M_{d} & \approx\left(\begin{array}{ccc}
0 & b^{\prime} \bar{\epsilon}^{3} & 0 \\
b^{\prime} \bar{\epsilon}^{3} & \bar{\epsilon}^{2} & a^{\prime} \bar{\epsilon} \\
0 & a^{\prime} \bar{\epsilon} & 1
\end{array}\right)
\end{aligned}
$$

where $a, a, b$ and $b^{\prime}$ are all of $O(1)$ and $S U(2)_{R}$ symmetry would imply $a=$ $a^{\prime} ; b=b^{\prime}$.

Diagonalising these mass matrices gives the results of eq(1) and 


$$
\begin{aligned}
\frac{m_{s}}{m_{b}} & =\left|\bar{\epsilon}^{2}\left(1-a^{2}\right)\right| \\
\frac{m_{d} m_{s}}{m_{b}^{2}} & =\left|b^{\prime 2} \bar{\epsilon}^{6}\right| \\
\frac{m_{c}}{m_{t}} & =\left|\epsilon^{2}\left(1-a^{2}\right)\right| \\
\frac{m_{u} m_{c}}{m_{t}^{2}} & =\left|b^{2} \epsilon^{6}\right| \\
\left|V_{c b}\right| & =\left(a^{\prime} \frac{m_{s}}{m_{b}}+a \frac{m_{c}}{m_{t}}+2 \sqrt{a a^{\prime} \frac{m_{s} m_{c}}{m_{b} m_{t}}} \cos \phi\right)^{\frac{1}{2}}
\end{aligned}
$$

where $\phi$ and $\phi^{\prime}$ are phases related to the phases of $\epsilon, \bar{\epsilon}, a, b, a^{\prime}$ and $b^{\prime}$. The predictions of eq(1) for $\left|V_{u b} / V_{c b}\right|$ and $\left|V_{u s}\right|$ follow from the texture zeros. The relation for $\left|V_{c b}\right|$ can be satisfied by a choice of $a^{\prime}$ and $a$ of $O(1)$. Unfortunately it does not prove that the solution has the larger $S U(2)_{R}$ symmetry with $a^{\prime}=a$ although it is clearly consistent with it. The quark mass ratios are successfully predicted up to the coefficients of $O(1)$ because the predictions

$$
\begin{aligned}
& \left(\frac{m_{s}}{m_{b}}\right)^{3} \approx \frac{m_{d} m_{s}}{m_{b}^{2}} \\
& \left(\frac{m_{c}}{m_{t}}\right)^{3} \approx \frac{m_{u} m_{c}}{m_{t}^{2}}
\end{aligned}
$$

hold for the observed quark masses (cf Table 2 ). If $S U(2)_{R}$ is a good symmetry we may derive from eq(16) an equality (at the unification scale)

$$
\frac{m_{d} m_{b}}{m_{s}^{2}}=\frac{m_{u} m_{t}}{m_{c}^{2}}
$$

After including radiative corrections this is consistent with present bounds.

Thus we conclude that the quark mass matrices are presently in excellent agreement with a very large underlying symmetry. This symmetry consists of left- right- symmetry together with a $S U(2)_{R}$ symmetry of Yukawa couplings and a $U(1)$ horizontal family symmetry. More detailed tests of this symmetry will be forthcoming with the improved precision on the determination of the quark mixing angles and the discovery of the top quark. As is evident from Table 2 the differences between the various solutions are significant and should be measurable.

\section{Lepton masses}

Let us now consider the structure of lepton masses resulting from the $U(1)_{F D}$ symmetry. The lepton-antilepton charge structure is given in eq(4). If we are to 
maintain the successful relation $m_{b} \approx m_{\tau}$ at the unification scale we must have $\alpha_{1}=a_{1}$. In this case the lepton mass matrix has the form

$$
M^{L} \approx\left(\begin{array}{ccc}
\bar{\epsilon}^{|-4-2 b|} & \bar{\epsilon}^{|-3|} & \bar{\epsilon}^{|-b-2|} \\
\bar{\epsilon}^{|-3|} & \bar{\epsilon}^{2(b-1) \mid} & \bar{\epsilon}^{|b-1|} \\
\bar{\epsilon}^{|-b-2|} & \bar{\epsilon}^{|b-1|} & 1
\end{array}\right)
$$

where $b=a_{2} / a_{1}$. Although we have no measured lepton mixing angles to guide us we will argue that even in the lepton sector there is evidence for the same texture zero structure as we have in the up and down quark matrices. The reason may be seen from the structure of eq(19) in which the $(1,2)$ and $(2,1)$ matrix elements are independent of the parameter $b$ and are the same as the equivalent down quark matrix elements. This means that if there are texture zeros in the $(1,1)$ and $(1,3)$ positions (corresponding, as discussed above, to the range $b>1$ ) we have the prediction $\operatorname{Det}\left(M_{d}\right)=\operatorname{Det}\left(M_{l}\right)$. As originally observed by Georgi and Jarlskog [16], this relation is in excellent agreement with the observed masses when continued to the unification scale for, together with the relation $m_{b}=m_{\tau}$, it leads to the result

$$
\begin{aligned}
& m_{d} m_{s} \approx m_{e} m_{\mu} \text { at the unification scale } \\
& m_{d} m_{s} \approx 9 m_{e} m_{\mu} \text { at laboratory energies }
\end{aligned}
$$

Thus the texture zero structure for leptons, which is predicted for $b>1$ by the anomaly free structure of $U(1)_{F D}$, generates the excellent mass prediction of eq(20).

To go further and determine $m_{\mu}$ and $m_{e}$ separately we need to specify the value of $b$. For $b=3$, the lepton charges are the same as the down quark sector, and so the structure of the down quark and lepton mass matrices are identical. However there is another choice of the charge ratio $b$ which does explain the structure of this sector. If $b=3 / 2$ the lepton mass matrix has the form

$$
M_{L}=\left(\begin{array}{ccc}
\bar{\epsilon}^{5} & \bar{\epsilon}^{3} & 0 \\
\bar{\epsilon}^{3} & \bar{\epsilon} & 0 \\
0 & 0 & 1
\end{array}\right)
$$

The zeros in this mass matrix result because with this choice for $b$, there is a residual $Z_{2}$ discrete gauge symmetry after $U(1)$ breaking by which the electron and muon fields get transformed by a factor $(-1)$. In this case we have the relations at laboratory energies

$$
\begin{aligned}
& m_{\mu} \approx \frac{m_{s}}{3 \bar{\epsilon}} \\
& m_{e} \approx \frac{m_{d} \bar{\epsilon}}{3}
\end{aligned}
$$

which are in good agreement with the values of Table 2 for the value $\bar{\epsilon}=0.23$ needed to fit the down quark masses and mixing angles. 


\section{Anomaly cancellation and the structure of $U(1)_{F I}$}

We have seen that a simple assignment of $U(1)_{F D}$ charges leads to predictions for the structure of the quark and lepton masses in remarkable agreement with experiment. However we have also noted that this is only possible if we assign charges to the Higgs fields which apparently introduce an $S U(2)^{2} U(1)$ anomaly. Here we discuss cancellation of anomalies for the case that a $U(1)_{F I}$ piece is added. We will show in this section that, while there are still anomalies, they are of the type which may be cancelled by the GS mechanism of string theory provided $\sin ^{2}\left(\theta_{W}\right)=3 / 8$ at the string scale.

By definition $U(1)_{F D}$ in eq(2) acting on the quarks and leptons is traceless and hence has vanishing $S U(3)^{2} U(1), S U(2)^{2} U(1)$ and $U(1)_{Y}^{2} U(1)$ anomalies]. Thus for an anomaly free solution we must choose $U(1)_{F I}$ to be anomaly free. With the minimal particle content of the MSSM, the only $U(1)$ s which are anomaly free and flavour independent are the weak hypercharge itself and a symmetry $U(1)_{H}$ which gives opposite charge to the two doublets. As we noted at the end of Section 4.2 this does not allow for viable mass matrices. However in theories derived from a string theory there is a significant new possibility for a non-vanishing anomaly associated with a new $U(1)$ gauge factor can be cancelled by the Green-Schwarz (GS) anomaly cancellation mechanism [9, 17].

In the 4-D version of the GS mechanism one cancels the anomalies of a single $U(1)$ by an appropriate shift of the axion present in the dilaton multiplet of fourdimensional strings. This happens because such an axion has a direct coupling to $F \tilde{F}$. For the GS mechanism to be possible, the coefficients $A_{i}, i=3,2,1$ of the mixed anomalies of the $U(1)$ with $S U(3), S U(2)$ and $U(1)_{Y}$ have to be in the ratio $A_{3}: A_{2}: A_{1}=k_{3}: k_{2}: k_{1}$ [18]. Here $k_{i}$ are the Kac- Moody levels of the corresponding gauge factors and they determine the boundary condition of the gauge couplings at the string scale by the well-known equation $g_{3}^{2} k_{3}=$ $g_{2}^{2} k_{2}=g_{1}^{2} k_{1}^{2}$. The usual (e.g. GUT) canonical values for these normalization factors (corresponding to the successful result $\sin ^{2}\left(\theta_{W}\right)=3 / 8$ ) yield $k_{3}: k_{2}$ : $k_{1}=1: 1: 5 / 3$ and hence the GS mechanism can only work in this case if the mixed anomalies of the $U(1)$ with the SM gauge factors are in the ratio $A_{3}: A_{2}: A_{1}=1: 1: 5 / 3[18]$. One can easily convince oneself that there are only two $U(1)$ symmetries with anomalies consistent with this ratio of gauge coupling constants, namely $U(1)_{X}$ and $U(1)_{X X}$ given in Table 1 .

Thus, in a supersymmetric SM coming from a string the most general familyindependent anomaly-free $U(1)$ consistent with canonical gauge coupling unification is given by:

$$
U(1)_{F I}=z U(1)_{H}+x U(1)_{X}+y U(1)_{X X} .
$$

The full charges of the $U(1)$ factor of eq(2) may now be determined using Tables

\footnotetext{
${ }^{7}$ We have use the freedom to define the Higgs $U(1)$ charges to be entirely in $U(1)_{F I}$.
} 


\begin{tabular}{|c|ccccccc|}
\hline & $\mathrm{Q}$ & $\mathrm{u}$ & $\mathrm{d}$ & $\mathrm{L}$ & $\mathrm{e}$ & $H_{2}$ & $H_{1}$ \\
\hline$U(1)_{H}$ & 0 & 0 & 0 & 0 & 0 & 1 & -1 \\
\hline$U(1)_{X X}$ & 0 & 0 & 1 & 1 & 0 & 0 & 0 \\
\hline$U(1)_{X}$ & 1 & 1 & 0 & 0 & 1 & 0 & 0 \\
\hline
\end{tabular}

Table 4: Anomaly-free $U(1)_{F I}$ symmetries.

\begin{tabular}{|c|ccccccc|}
\hline & $\mathrm{Q}$ & $\mathrm{u}$ & $\mathrm{d}$ & $\mathrm{L}$ & $\mathrm{e}$ & $H_{2}$ & $H_{1}$ \\
\hline$U(1)$ & $\alpha_{i}+x$ & $\alpha_{i}+x$ & $\alpha_{i}+y$ & $a_{i}+y$ & $a_{i}+x$ & $\mathrm{z}-2 \alpha_{1}$ & $-\mathrm{z}+2 \alpha_{1}$ \\
\hline
\end{tabular}

Table 5: Anomaly-free $U(1)$ symmetries.

3 and 4 and give the charges shown in Table $50^{8}$.

The choice $z=-2 x$ gives the results of eq(可) for the up quark mass matrix. If one further has $3 x+y=-4 \alpha_{1}$ one gets the results of eq(11) for the d-quark masses. Note, however, the choice of the flavour-independent component allows for further possibilities for the down quark matrices. In particular, the alternative given by eq(12) may be obtained if $3 x+y=-7 / 2 \alpha_{1}$. We conclude that the generic problem raised by anomaly cancellation may naturally be solved in the context of string based models.

So far we have assumed that $\sin ^{2}\left(\theta_{W}\right)=3 / 8$ at the string unification scale. In fact an acceptable pattern of fermion masses actually requires this value! To see this let us compute the mixed anomalies $A_{i}$ for the $U(1)_{F D}$ symmetry of Table 3 with $S U(3), S U(2)$ and $U(1)_{Y}$. One finds respectively:

$$
\begin{aligned}
& A_{3}=2 \sum_{i=1}^{3} \alpha_{i} \\
& A_{2}=\frac{3}{2} \sum_{i=1}^{3} \alpha_{i}+\frac{1}{2} \sum_{i=1}^{3} a_{i}+\frac{\alpha_{1}}{2}(w-2) \\
& A_{1}=\frac{11}{6} \sum_{i=1}^{3} \alpha_{i}+\frac{3}{2} \sum_{i=1}^{3} a_{i}+\alpha_{1}(w-2)
\end{aligned}
$$

where, as we are working with the full $U(1)$ charges, we no longer have $\alpha_{3}=$ $-\left(\alpha_{1}+\alpha_{2}\right)$ and $a_{3}=-\left(a_{1}+a_{2}\right)$ as the $U(1)_{F I}$ piece adds a family independent term to $\alpha_{i}$ and also to $a_{i}$. However, to maintain the result $m_{b}=m_{\tau}$ we have $a_{1}=\alpha_{1}$. Furthermore, the texture zeros together with the result $\operatorname{Det}\left(M_{L}\right)=\operatorname{Det}\left(M_{d}\right)$ requires $a_{2}+a_{3}=\alpha_{2}+\alpha_{3}$. Thus the requirement of an acceptable mass pattern gives the constraint:

\footnotetext{
${ }^{8}$ The terms proportional to $2 \alpha_{1}$ in $H_{1,2}$ could be absorbed in $\mathrm{z}$.
} 


\begin{tabular}{|c|ccccccccc|}
\hline$U(1)_{F}$ & $\mathrm{Q}$ & $\mathrm{u}$ & $\mathrm{d}$ & $\mathrm{L}$ & $\mathrm{e}$ & $H_{1}$ & $H_{2}$ & $\theta$ & $\theta$ \\
\hline 3rd generation & 0 & 0 & 0 & 0 & 0 & 0 & 0 & 1 & -1 \\
2nd generation & 1 & 1 & 1 & $1 / 2$ & $1 / 2$ & & & & \\
1st generation & -4 & -4 & -4 & $-7 / 2$ & $-7 / 2$ & & & & \\
\hline
\end{tabular}

Table 6: Anomaly-free $U(1)$ gauge symmetry giving rise to the textures in $\operatorname{eqs}(7,11,21)$

$$
\sum_{i=1}^{3} \alpha_{i}=\sum_{i=1}^{3} a_{i}
$$

From eq(24) and eq(25) we see that the condition $A_{3}=A_{2}$, which is needed if the $S U(3)$ and $S U(2)$ couplings are unified, gives

$$
\begin{array}{r}
w=2 \text { or } \alpha_{1}=0 \\
\text { and } A_{3}: A_{2}: A_{1}=1: 1: \frac{5}{3}
\end{array}
$$

The second equation requiring $\sin ^{2}\left(\theta_{W}\right)=3 / 8$ may be seen as a consequence of an acceptable mass matrix structure. The first condition in eq(27) requires $w=2$ since $\alpha_{1}=0$ does not lead to an acceptable mass pattern.

To summarise this section we have seen that a simple $U(1)_{F D}$ extension of the standard model generates much of the quark and lepton mass matrix structure but that anomaly cancellation requires a $U(1)_{F I}$ component. Moreover the full $U(1)$ extension, including the Higgs doublets needed for the MSSM, is only anomaly free in the context of string theory via a Green Schwarz term. The charges of each individual particle with respect to this anomaly-free $U(1)$ giving rise to the favoured solution eqs([), (11) and (21) are shown in Table 6.

The simplicity of the assignments is remarkable. It is also worth emphasizing that this $U(1)$ symmetry may be made anomaly free through the GS mechanism if and only if the normalization of the coupling constants is the canonical one $g_{3}^{2}=$ $g_{2}^{2}=5 / 3 g_{1}^{2}$ yielding the succesfull prediction $\sin ^{2} \theta_{W}=3 / 8$. Thus the present scheme not only predicts a succesfull pattern of fermion masses and mixings but also predicts $\sin ^{2} \theta_{W}=3 / 8$ even without any grand unification group.

It is important also to recall that the $U(1) \mathrm{s}$ whose anomalies are cancelled through a GS mechanism are necessarily spontaneously broken not far below the string scale. The reason for this is that the piece in the Lagrangian cancelling the anomalies has a supersymmetric counterpart which is a sort of field-dependent Fayet-Iliopoulos term for the $U(1)$. This term forces $U(1)$ symmetry breaking in a natural way at a scale of order $1 / \sqrt{192} \pi M_{\text {string }}[19,20]$ Thus the present scheme also explains why the extra $U(1)$ symmetry required to generate the fermion mass patterns does not survive down to low energies. 
We close this section with two comments about the consistency of our solution with the other symmetries needed to build a viable supersymmetric theory. The first concerns the $\mu$ problem. This refers to the necessity to explain why the Higgs scalars $H_{1}$ and $H_{2}$ needed in the MSSM are light even though the mass term $H_{1} H_{2}$ is $S U(3) \otimes S U(2) \otimes U(1)$ invariant and hence would naturally be expected to be large. The problem can be solved by a discrete (gauge) symmetry unbroken down to the electroweak scale. It is straightforward to show that the smallest discrete symmetry capable of eliminating these terms is a $Z_{3}$ symmetry which, however, necessarily is not flavour blind in the lepton sector 21. Indeed it allows all possible Higgs couplings to quarks but the $(1,3)$ and $(2,3)$ couplings to leptons are forbidden. Clearly this symmetry is consistent with the form of eq(21) which followed from the choice $b=3 / 2$.

The second comment concerns the possible baryon and lepton number violating terms. The $U(1)$ symmetry determining the structure of masses does not forbid the presence of R-parity violating terms like $t_{R} b_{R} s s_{R}$ or $t_{L} b_{L}^{c} \tau_{L}$, it was not designed to do that. If such terms are to be suppressed there must be an extra discrete symmetry doing the job. Alternatively, one can try to extend the above $U(1)$ to forbid all R-parity violating terms. Adding to the $U(1)_{F I}$ symmetry one piece proportional to the third component of right-handed weak isospin $U(1)_{R}$ may be useful in this respect. If this is done, one has to be careful so that a residual discrete $Z_{N}$ gauge symmetry survives doing the job after $U(1)$ symmetry breaking.

\section{Summary and Conclusions.}

To summarise, eqs(17), (11) and (21) determine the order of magnitude of the 12 quark and lepton masses and mixing angles in terms of just four parameters, $|\epsilon|$, $|\bar{\epsilon}|$ together with the top Yukawa coupling, $h_{t}$ and the ratio of Higgs vevs given in terms of the usual parameter $\tan \beta$. Adding the requirement that the radiative correction should give an acceptable b quark mass reduces this essentially to just three for $m_{t} \geq 170 \mathrm{Gev}$ is required to keep $m_{b}$ from becoming too large 1 . In addition, consistency with this fermion mass structure yields the successful prediction $\sin ^{2}\left(\theta_{W}\right)=3 / 8$ at the unification scale.

If we wish to determing the precise magnitude of the 13 masses, mixing angles and $\mathrm{CP}$ violating phase, the most symmetric solution has the eight parameters of eqs(14), (15) and (21), $\left|1-a^{2}\right|,|b|,|\epsilon|,|\bar{\epsilon}|, \phi, \phi^{\prime}$, together with the top Yukawa coupling, $h_{t}$ and the ratio of Higgs vevs given in terms of the usual parameter $\tan \beta$. This is consistent with a very large underlying symmetry given by a horizontal U(1) family symmetry constrained by anomaly cancellation, leftright- symmetry giving symmetric mass matrices, $S U(2)_{R}$ symmetry relating up and down quark couplings and a down- quark lepton symmetry.

We find it quite remarkable that the apparently complicated pattern of quark and lepton masses may be explained by a very simple flavour and family symmetry 
of the type discussed here. The fact that a suitable choice of symmetry plus multiplet structure is sufficient to fix a phenomenologically realistic pattern of masses and mixing angles demonstrates how the problem of understanding the latter may be transformed into the problem of determining the former. In 4-D string theories the symmetries and multiplet structure are just the things that are expected in a definite string compactification. What our analysis shows is that the symmetries of the Yukawa couplings may be significant and point to an underlying unification certainly consistent with the expectation in string theory. Of course the next step is to identify the "correct" 4-D string theory, but that is another story!

\section{References}

[1] G. Costa, J. Ellis, G.L. Fogli, D.V. Nanopolous and F. Zwirner, Nucl.Phys. B297 (1988) 244;J. Ellis, S. Kelley and D.V. Nanopoulos, Phys. Lett. B249 (1990)441;Phys. Lett. B260 (1991) 131; P. Langacker, Pennsylvania preprint UPR-0435T, (1990); U. Amaldi, W. de Boer and H. Fürstenau, Phys. Lett. B260 (1991) 447;P. Langacker and M. Luo, Phys.Rev.D44 (1991) 817; G.G. Ross and R.G. Roberts, Nucl. Phys. B 377 (1992) 571; F.Anselmo, L. Ciafarelli, A. Peterman and A. Zichichi, Nuovo Cim. 104A (1991) 1817 and CERN preprint CERN-TH.6429/92.

[2] K. Inoue et al., Prog.Theor.Phys. 68 (1982) 927; L.E. Ibáñez, Nucl.Phys. B218 (1983) 514; L.E. Ibáñez and C. López, Phys. Lett. B126 (1983) 54; Nucl.Phys. B233 (1984) 511; L. Alvarez-Gaume, J. Polchinsky and M. Wise, Nucl.Phys. B221 (1983) 495; L.E Ibáñez, C. López and C. Muñoz, Nucl. Phys. B256 (1985) 218.

[3] M.S. Chanowitz, J. Ellis and M.K. Gaillard, Nucl. Phys. B 128 (1977) 506. A. Buras, J. Ellis, M.K. Gaillard and D.V. Nanopoulos, Nucl. Phys. B 135 (1978) 66.

[4] H. Arason, D.J. Castaño, B. Keszthelyi, S. Mikaelian, E.J. Piard, P. Ramond and B.D. Wright, Phys. Rev. Lett. 67 (1991) 2933.

[5] S. Weinberg, in "A Festschrift for I.I. Rabi" [Trans. N.Y. Acad. Sci., Ser. II (1977), v. 38], p. 185; F. Wilczek and A. Zee, Phys. Lett. B 70 (1977) 418. T. Maehara and T. Yanagida, Prog. Theor. Phys. 60 (1978) 822 J. Chakrabarti, Phys. Rev. D20 91979) 2411 F. Wilczek and A. Zee, Phys. Rev. Lett. 42 (1979) 421

[6] H. Fritsch,Phys. Lett. B 70 (1977) 436; Phys. Lett. B 73 (1978) 317; F.J. Gilman and Y. Nir, Ann. Rev. Nucl. Part. Sci. 40 (1990) 213; P. Kaus and S. Meshkov, Mod. Phys. Lett. A3(1988) 1251. 
[7] C.D.Froggat and H.B. Nielsen, Origin of symmetries, World Scientific (1991) N. Cabibbo, Phys. Rev. Lett. 10 (1963) 531 M. Kobayashi and T. Maskawa, Prog. Theor. Phys. 49 (1973) 652 M.E. Machacek and M.T. Vaughn, Phys. Lett. B103 (1981) 427

[8] J. Harvey, P. Ramond and D. Reiss, Phys. Lett. B 92 (1980) 309; S. Dimopoulos, L.J. Hall and S. Raby, Phys. Rev. Lett. 68 (1992) 1984; Phys. Rev. D 45 (1992) 4195; H. Arason, D.J. Castaño, P. Ramond and E.J. Piard, Phys. Rev. D 47 (1993) 232; G.F. Giudice, Mod. Phys. Lett. A7 (1992) 2429 .

[9] For a review of string theories, see M. Green, J. Schwarz and E. Witten, Superstring Theory, Cambridge University Press, 1987.

[10] A. Buras and M.K. Harlander, Munich preprint MPI- PAE/PTh 1/92; J.L. Rosner, Jour. Phys. G18(1992) 1575.

[11] P.Ramond, R.G.Roberts and G.G.Ross, Nucl. Phys. B406(1993)19

[12] C.D.Froggart and H.B.Nielsen, "Origin of Symmetries", World Scientific 1991.

[13] M.Leurer, Y.Nir and N.Seiberg, Nucl.Phys.B398(1993)319;Rutgers preprint RU-93-43, WIS-93/93/Oct- PH(1993) Y.Nir and N.Seiberg, Phys.Lett. B309(1993)337

[14] C.D. Froggatt and H.B. Nielsen, Nucl. Phys. B147 91979) 277 C.D. Froggatt and H.B. Nielsen, Nucl. Phys. B164 (1979) 144 S. Dimopoulos, Phys. Lett. B129 (1983) 417 G. Anderson et al, lawrence Berkeley Lab Preprint LBL33531, UCB- PTH-93/03 (1993)

[15] H. Fritzsch and J. Plankl,Phys. Lett. B 237 (1990) 451 and references therein.

[16] H. Georgi and C. Jarlskog, Phys. Lett. B 86 (1979) 297.

[17] M. Green and J. Schwarz, Phys. Lett. B 149 (1984) 117

[18] L.E. Ibáñez, Phys. Lett. B 303 (1993) 55

[19] M. Dine, N. Seiberg and E. Witten, Nucl.Phys. B289 (1987) 585; J.Atick, L. Dixon and A. Sen, Nucl.Phys. B292 (1987) 109; M. Dine, I. Ichinoise and N. Seiberg, Nucl.Phys. B293 (1987) 253.

[20] A. Font, L.E. Ibáñez, H.P. Nilles and F. Quevedo, Nucl.Phys. B307 (1988) 109; Phys. Lett. B 210 (1988) 101; J.A. Casas, E.K. Katehou and C. Muñoz, Nucl.Phys. B317 (1989) 171; J.A. Casas and C. Muñoz, Phys. Lett. B 209 (1988) 214 ;Phys. Lett. B 214 (1988) 63; A. Font, L.E. Ibáñez, F. Quevedo and A. Sierra, Nucl.Phys. B331 (1990) 421. 
[21] L.E.Ibanez and G.G.Ross, in preparation. 